Research Paper

\title{
The Efficacy and Safety of First-line Chemotherapies for Advanced Biliary Tract Cancer: A Network Meta-analysis
}

Wei Zheng1* ${ }^{*}$ Jie Ying $3^{3 *}$, Yan Zhou ${ }^{2}$, Zhiwen Lu², Ke Min², Weimin Wang2, Yun Zhang1, Miao Zhang', Jian Yang $2^{\bowtie}$

1. Department of General Surgery, the Affiliated Yixing Hospital of Jiangsu University, Yixing, Jiangsu 214200, China.

2. Department of Oncology, the Affiliated Yixing Hospital of Jiangsu University, Yixing, Jiangsu 214200, China.

3. Department of Clinical Research Center, Xuyi People's Hospital, Xuyi, Jiangsu 211700, China.

*These authors contributed equally to this work.

$\triangle$ Corresponding author: Dr Jian Yang, Department of Oncology, The Affiliated Yixing Hospital of Jiangsu University, Tongzhenguan Road No.75, Yixing 214200, Jiangsu Province, China. Tel: +86 0510 87330792; Fax: +86 0510 87330791; E-mail: staff1661@yxph.com

(C) Ivyspring International Publisher. This is an open access article distributed under the terms of the Creative Commons Attribution (CC BY-NC) license (https://creativecommons.org/licenses/by-nc/4.0/). See http://ivyspring.com/terms for full terms and conditions.

Received: 2018.05.26; Accepted: 2018.10.28; Published: 2019.01.01

\begin{abstract}
Major chemotherapeutic drugs for advanced biliary tract cancer (ABTC) include gemcitabine, fluoropyrimidines and platinum compounds, but the optimum combination of them remains inconclusive. The main objective of this network meta-analysis was to compare the efficacy and safety of first-line chemotherapies for ABTC.

Methods: We searched PubMed, EMBASE, the Cochrane library and Science Direct for relevant controlled trials until May 2017. We estimated the Hazard ratios (HRs) for survival time and odds ratios (ORs) for response rate and toxic effects among different therapies. All data were calculated by Aggregate Data Drug Information System (ADDIS) v2.0 online and STATA software.

Results: 16 trials involving 2245 patients and 10 regimens were included in this study. In terms of the objective response rate, Cap plus CIS (CapC) exhibited better performance than FU (OR 5.46, 95\% Cl 1.07-56.63). Gem plus S-1 (GS) was superior to Gem (OR 4.72, 95\% Cl 1.31-17.02) and FU (OR 9.08, $95 \% \mathrm{Cl} 1.56-89.20)$. Also, GS had an overall survival benefit compared to FU and Gem, with a HR of 0.51 $(95 \% \mathrm{Cl} 0.28-0.96)$ and $0.43(95 \% \mathrm{Cl} 0.20-0.93)$, respectively. Compared with FU, Gem plus OXA (Gemox) prolonged the OS (HR 0.57, 95\% Cl 0.32-0.96). And FU was also inferior to FP (HR 1.88, 95\% $\mathrm{Cl}$ 1.07-3.16). The PFS did not differ between all regiments. The incidence of grade 3 or 4 hematological toxic effects appeared to be higher in the Gem-based chemotherapies. In regard to nonhematological adverse events, grade 3 or 4 diarrhea and stomotitis occurred more frequently in S-1-based groups. In addition, the Cap plus CIS combination (CapC) were more likely to cause vomiting, stomotitis and hand-foot syndrome. As for peripheral neuropathy, Gem plus OXA (Gemox), CapC and GC were associated with higher risk. There was no difference among different treatments with respect to anorexia, fatigue, nausea, pigmentation, renal dysfunction and asthenia.
\end{abstract}

Conclusion: Physicians should discuss with the patients the different options outlining potential benefit and toxicity since no clear evidence of an approach of choice can be produced.

Key words: advanced biliary tract cancer, chemotherapy, gemcitabine, fluoropyrimidines, platinum compounds, network meta-analysis

\section{Introduction}

Biliary tract cancer (BTC) arises from the biliary epithelium of the small ducts in the periphery of the liver (intrahepatic) and the main ducts of the hilum (extrahepatic). Extrahepatic biliary tract cancers include gallbladder cancer, ampullary cancer, and cancer of the pancreatic biliary ducts [1]. These 
cancers are relatively rare, but the annual incidence and mortality of BTC are increasing worldwide, with the highest rates in Latin American and Asian populations [2]. Although surgery is currently the only curative treatment of BTC, the majority $(80 \%)$ of patients have locally advanced or distant metastatic disease at presentation [3]. The prognosis of unresectable BTC is aggressive, and less than $10 \%$ of patients are alive at 5 years after diagnosis [4]. Therefore, palliative therapy is important for patients with advanced biliary tract cancer (ABTC).

Current National Comprehensive Cancer Network (NCCN) practice guidelines endorse gemcitabinecisplatin combination chemotherapy as a category 1 recommendation for the first-line treatment of patients with $\mathrm{ABTC}$, on the basis of $\mathrm{ABC}-02$ trail [5]. In the $\mathrm{ABC}-02$ trial, gemcitabine-cisplatin significantly improved overall survival (OS), progression-free survival (PFS), and disease control rate (DCR) compared with gemcitabine monotherapy. However, the prognosis remains poor, with a median survival time less than 1 year (11.7 month) [6, 7]. Therefore, new drugs and combination therapies for BTC are highly demanded.

Target-specific monoclonal antibodies and small molecules inhibitors directed against the signaling pathways that drive BTC growth and invasion have been developed [8]. As we understand more about the molecular pathways involved in BTC, targeted therapies are at the forefront of new therapeutic combinations. However, targeted therapies combined with chemotherapy have failed or shown only marginal benefits in several clinical trials. Nowadays, we mainly improve prognosis of $\mathrm{ABCT}$ by using chemotherapies. Gemcitabine (Gem), fluoropyrimidines (fluorouracil (FU), S-1, capecitabine (Cap) and tegafur et al), and platinum compounds (cisplatin (CIS), oxaliplatin (OXA) et al) are considered key drugs for the treatment of ABTC. Lots of controlled trials have been conducted to explore the optimum combination of these regimens, and yet no conclusive results have been reached. Therefore, we conducted a Bayesian network meta-analysis to assess the efficacy and safety of different chemotherapy regimens directly or indirectly in patients with ABTC.

\section{Methods}

\section{Search strategy}

PubMed, EMBASE, the Cochrane library and Science Direct were retrieved, with a censor date up to September 2017. Following terms were used in the searching procedure: (advanced biliary tract cancer/ carcinoma/neoplasms OR cholangiocarcinoma) AND (chemotherapy). The search was limited to English language and human-based papers. To ensure that all relevant trials were included, we scanned related literature and references in the selected articles.

\section{Study selection}

We checked every paper by viewing the title, abstract, and the full text. Eligible studies were controlled trials involving patients with ABTC who were treated with first-line chemotherapy. Studies involving the followings were excluded: targeted therapy, hepatic artery infusional chemotherapy, intraperitoneal chemotherapy, immunotherapy, combination therapy with a local control therapy such as palliative surgery, radiotherapy, or photodynamic therapy. We defined ABTC as unresectable or recurrent or metastatic disease. We distinguish monotherapy (single agent) and multidrug regimens by the number of effective components in the chemotherapy regimen. For example, S-1 (also named Tiji'aoJiaonang in China) is a novel oral derivative of 5-FU, and contains tegafur / gimeracil / oteracil potassium in a molar ratio of 1.0:0.4:1.0. Tegafur (FT) is a depot form of fluorouracil, which releases 5-fluorouracil (5-FU) slowly in the body. Gimeracil, a dihydropyrimidine dehydrogenase inhibitor, contributes to a decrease in 5-FU catabolism and to significantly higher blood levels of 5-FU compared to FT alone. Oteracil potassium (Oxo), another enzyme inhibitor of 5-FU, can suppress the gastrointestinal toxicity of FT. FT has antitumor effect. Gimeracil and oteracil are biology regulators, but not chemotherapy agents. When used gimeracil or oteracil alone, there was no effect on the tumor. Accordingly, S-1 is considered as a single drug, not a combination chemotherapy regimen in clinical context. All different opinions were discussed.

\section{Data extraction}

Characteristics of studies, such as author name, year of publication, therapy regimens, number of each intervention, follow-up time were recorded exactly. The details of the efficacy and safety outcome, including objective response rate (ORR), overall survival (OS), progression-free survival (PFS) and adverse events (AEs) were captured respectively. The ORR was the percentage of patients who had a complete or partial tumor response. OS was defined as the time from random assignment to the date of death from any cause. PFS was calculated from the date of randomization to the date of disease progression or death from any cause. Original data was transformed into the forms suitable for meta-analysis where necessary. The above data extraction was performed by two reviewers independently. 


\section{Statistical Analysis}

We performed the multi-treatment meta-analysis within a Bayesian framework by Markov Chain Monte Carlo simulation. All data was calculated by using Aggregate Data Drug Information System (ADDIS) v2.0 online and STATA (Version 12.0; StataCorp, College Station, TX).

Hazard ratios (HRs) and 95\% confidence intervals (CIs) were used as effect indicators for time-toevent data (OS and PFS). Dichotomous data (ORR and AEs) was analyzed using odds ratio (OR) and 95\% CIs.

Statistical heterogeneity between studies was assessed with $\mathrm{I}^{2}$ statistics and $\mathrm{P}$ value. Values of $\mathrm{I}^{2}$ above $50 \%$, between $25 \%$ and $50 \%$, and lower than $25 \%$ were regarded respectively as of high, moderate, and low heterogeneity $[9,10]$. A random-effects model was used to incorporate direct and indirect data into a single comparison, if heterogeneity existed $\left(\mathrm{P}<0.05\right.$, or $\left.\mathrm{I}^{2}<25 \%\right)$ and could not be explained or corrected.

Inconsistency refers to the differences between direct and various indirect effects estimated for the same comparison. Node-splitting analysis is an alternative method to evaluate inconsistency by assessing whether direct and indirect evidences on the split node are in agreement. $\mathrm{P}<0.05$ was considered as significant heterogeneity. We also investigated possible sources of inconsistency using inconsistency factor (IF) among studies in each closed loop. If the 95\% CIs of IF values are including zero, it indicates that there is no significant inconsistency.
The publication bias was evaluated by a 'comparison-adjusted' funnel plots whose horizontal axis represented the difference between study-specific effect sizes and the corresponding comparisonspecific summary effect.

We further calculated ranking probabilities for each treatment's efficacy under different endpoint to provide basis for selection of alternatives.

\section{Results}

\section{Study characteristics}

After applying the inclusion and exclusion criteria, 16 relevant articles involving a total of 2245 patients were finally screened out. Figure 1 shows the flow chart of selection process. Eleven different therapeutic regimens with single or combined use of six drugs were obtained. Other basic characteristics of the included studies were presented in Table 1.

\section{Heterogeneity analysis, inconsistency tests and Publication bias}

In the pair-wise meta-analysis, $\mathrm{I} 2$ values for the comparisons of GC and CapC in OS (I2 = 0\%) and PFS $\left(\mathrm{I}^{2}=0 \%\right), \mathrm{GC}$ and Gem in ORR $\left(\mathrm{I}^{2}=0 \%\right)$, PFS $\left(\mathrm{I}^{2}=0 \%\right)$ and OS $\left(\mathrm{I}^{2}=0 \%\right)$ were less than $25 \%$. These results indicated that heterogeneity was low overall. And we presented the results of inconsistency tests, which did not indicate inconsistencies between the direct and indirect evidence for any of the efficacy and toxic safety outcomes. No significant publication bias was found in this meta-analysis as the funnel plots were symmetrical near the zero line (Figure 2).

Table 1. Main characteristics of included studies.

\begin{tabular}{|c|c|c|c|c|c|c|c|}
\hline Author & Year & $\begin{array}{l}\text { Follow-up } \\
\text { time (months) }\end{array}$ & Outcomes & Site & Stage & $\begin{array}{l}\text { No. of } \\
\text { patients }\end{array}$ & Comparison \\
\hline Anant Ramaswamy & 2017 & 36 & ORR, OS, PFS, AEs & gallbladder cancer & inoperable/metastatic & 326 & GC vs. Gemox \\
\hline Naminatsu Takahara & 2017 & 40 & ORR, OS, PFS, AEs & biliary tract cancer & inoperable/metastatic/recurrent & 212 & GS vs. GC \\
\hline Kwonoh PARK & 2017 & 30 & ORR, OS, PFS & biliary tract cancer & inoperable/metastatic/recurrent & 124 & GC vs. CapC \\
\hline Tulay Kus & 2017 & 44 & ORR & biliary tract cancer & inoperable/metastatic & 80 & GC vs. FP \\
\hline Jieun Lee & 2015 & 24 & ORR, PFS, AEs & biliary tract cancer & inoperable/metastatic/recurrent & 93 & GC vs. CapC \\
\hline Sang Myung Woo & 2013 & 40 & ORR, OS, AEs & biliary tract cancer & inoperable/metastatic/recurrent & 292 & GC vs. CapC \\
\hline Chigusa Morizane & 2013 & 24 & ORR, OS, PFS, AEs & biliary tract cancer & inoperable/metastatic/recurrent & 90 & GS vs. S-1 \\
\hline Takashi Sasaki & 2013 & 24 & ORR, OS, PFS, AEs & biliary tract cancer & inoperable/metastatic/recurrent & 62 & GS vs. Gem \\
\hline Park J M & 2013 & Not given* & ORR & biliary tract cancer & inoperable/metastatic & 54 & GC vs. Gem \\
\hline Myoung Joo Kang & 2012 & 30 & ORR, OS, PFS, AEs & biliary tract cancer & inoperable/metastatic & 88 & CS vs. GC \\
\hline Juan Valle & 2010 & 32 & ORR, OS, PFS, AEs & biliary tract cancer & inoperable/metastatic & 303 & GC vs. Gem \\
\hline T Okusaka & 2010 & 24 & ORR, OS, PFS, AEs & biliary tract cancer & inoperable/metastatic/recurrent & 83 & Gem vs. GC \\
\hline Atul Sharma & 2010 & 28 & ORR, OS, PFS, AEs & gallbladder cancer & inoperable & 54 & FU vs. Gemox \\
\hline Mi-Jung Kim & 2008 & 48 & ORR, OS, PFS & biliary tract cancer & inoperable/metastatic/recurrent & 177 & GC vs. FP \\
\hline Naohiro Yonemoto & 2007 & 48 & ORR & biliary tract cancer & inoperable & 152 & $\begin{array}{l}\text { FU vs. S-1 vs. Gem } \\
\text { vs. Cisplatin }\end{array}$ \\
\hline M. Ducreux & 2005 & 38 & ORR, OS, PFS, AEs & biliary tract cancer & inoperable/metastatic & 55 & FU vs. FP \\
\hline
\end{tabular}

Note 1: GC=gemcitabine+cisplatin; Gemox=gemcitabine+oxaliplatin; GS=gemcitabine+S-1; CapC=capecitabine+ciaplatin; FP=5-FU+cisplatin; Gem=gemcitabine;

$\mathrm{CS}=$ cisplatin+S-1; FU=5-FU. Note 2: *Only objective response rate was showed in this article, the survival time and follow-up time were not reported 


\begin{tabular}{l} 
Potentially relevant articles identified from: \\
Pubmed (1912) \\
EMBASE (1083) \\
Science Direct (2433) \\
The Cochrane library (93) \\
\hline
\end{tabular}

The Cochrane library (93)

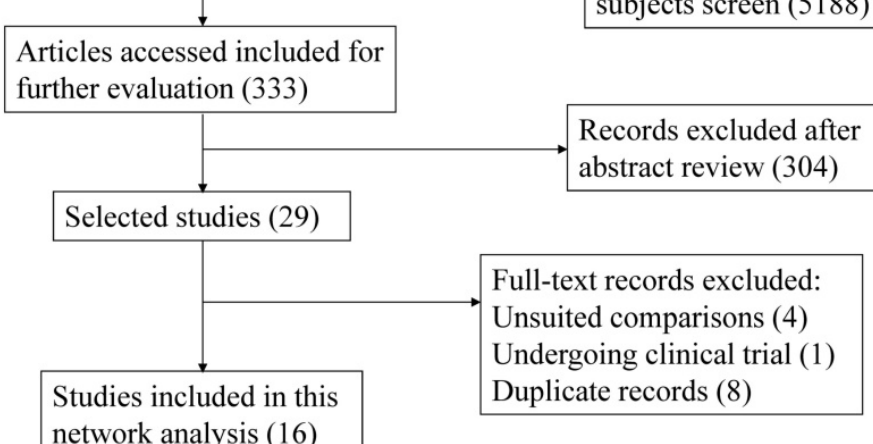
network analysis (16)

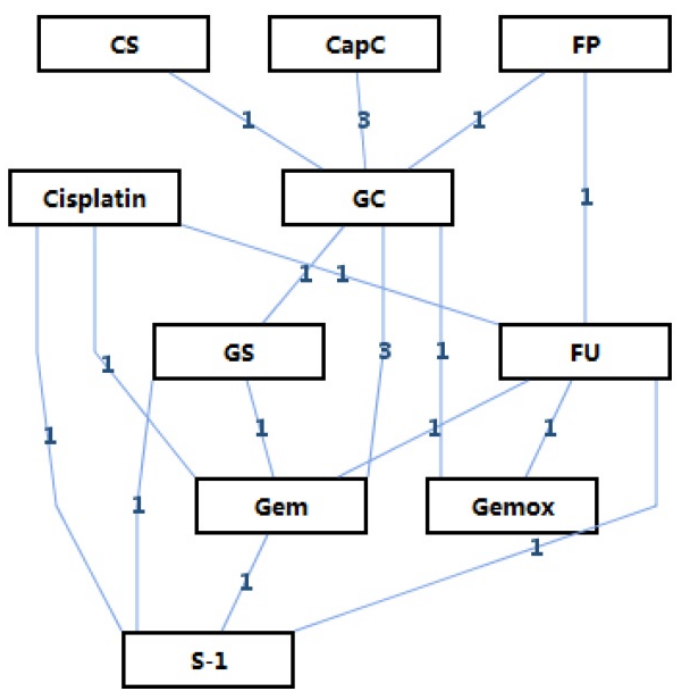

Figure 1. Flow chart and network structure.

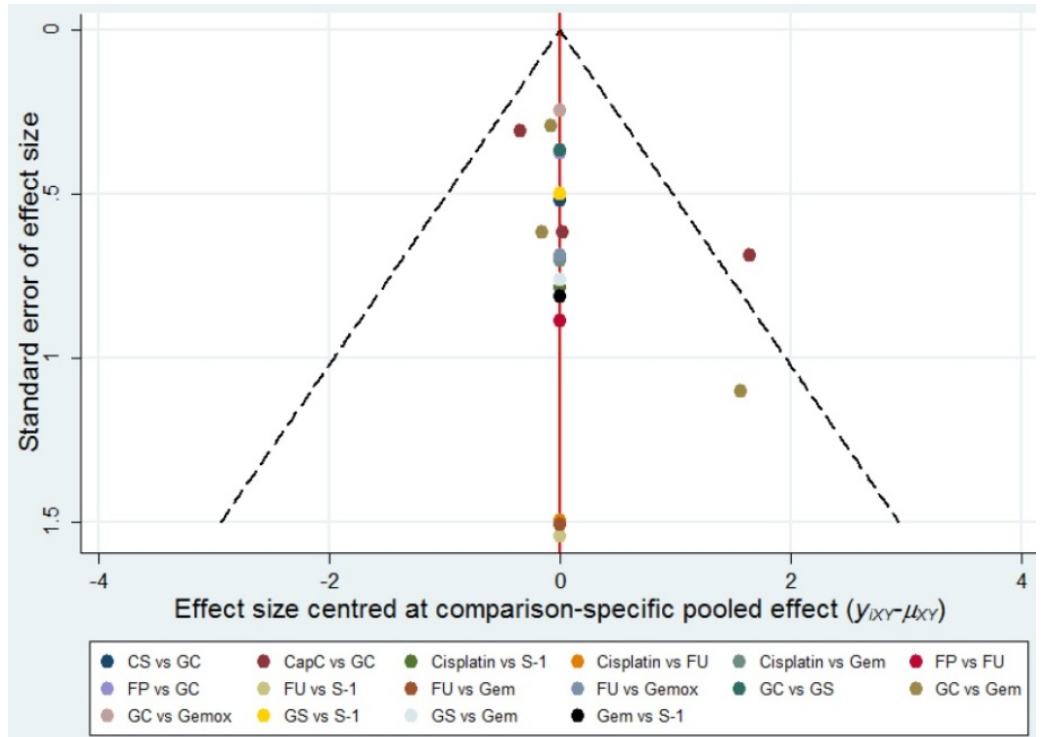

Figure 2. Publication bias. The red line represents the null hypothesis that the study-specific effect sizes do not differ from the respective comparison-specific pooled effect estimates. 


\section{ORR}

As presented in Table 2, Gem plus S-1 (GS) was superior to both Gem (OR 4.72, 95\% CI 1.31-17.02) and FU (OR 9.08, 95\% CI 1.56-89.20). Cap plus CIS (CapC) exhibited better performance than FU (OR 5.46, 95\% CI 1.07-56.63). We did not detect significant difference among other treatments.

\section{OS and PFS}

According to the results of network analysis, GS had an overall survival benefit compared to FU and Gem, with a HR of 0.51 (95\% CI 0.28-0.96) and 0.43 (95\% CI 0.20-0.93), respectively. Compared with FU, Gem plus OXA (Gemox) prolonged the OS (HR 0.57, 95\% CI 0.32-0.96). And FU was also inferior to FP (HR $1.88,95 \%$ CI 1.07-3.16). The PFS did not differ between these regiments. The details are listed in Table 2.

\section{Adverse Events (AEs)}

The incidence of grade 3 or 4 hematological toxic effects appeared to be higher in the Gem-based chemotherapies. Gem plus CIS (GC) and GS lead to more leucopenia and febrile neutropenia events compared with CS. The risk of anemia and neutropenia were higher with GC than CS or Gem. In

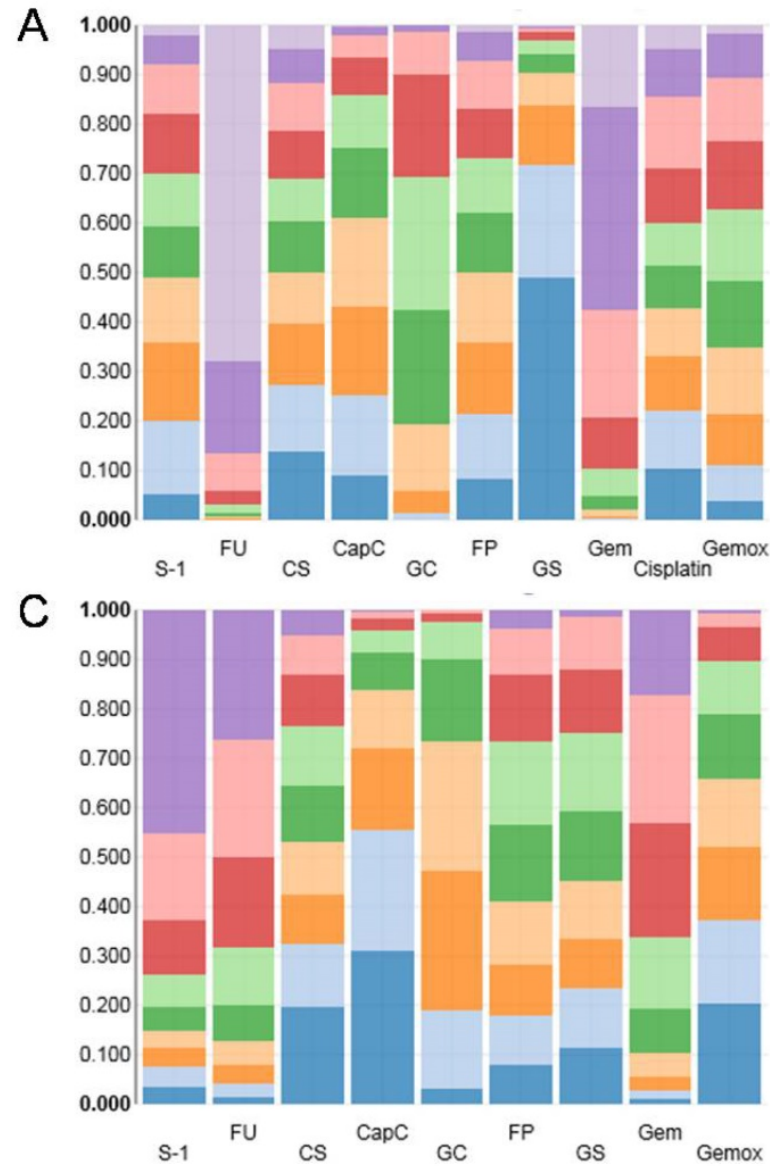

regard to nonhematological adverse events, grade 3 or 4 diarrhea and stomotitis occurred more frequently in S-1-based groups. The Cap plus CIS combination (CapC) were more likely to cause vomiting, stomotitis and hand-foot syndrome. In terms of peripheral neuropathy, Gem plus OXA (Gemox), CapC and GC were associated with higher risk. No difference was identified among treatments with respect to anorexia, fatigue, nausea, pigmentation, renal dysfunction and asthenia (Table 3).

\section{Ranking of treatments}

The results of rank probability were presented in Figure 3 and Figure 4. From the ranking of efficacy outcomes, GS, CS and CapC presented a high probability at ORR. CapC, CS and Gemox were outstanding PFS terms. And GS, FP and CS were the top three effective therapies with respect to OS. However, GC, GS, CapC, Gemox were associated with high risk of adverse events. On the other hand, due to the lack of data in compare of FP with other regiments, its toxic effects were uncertain. It appeared that there was no ideal treatment which can perform well in both efficacy and safety.
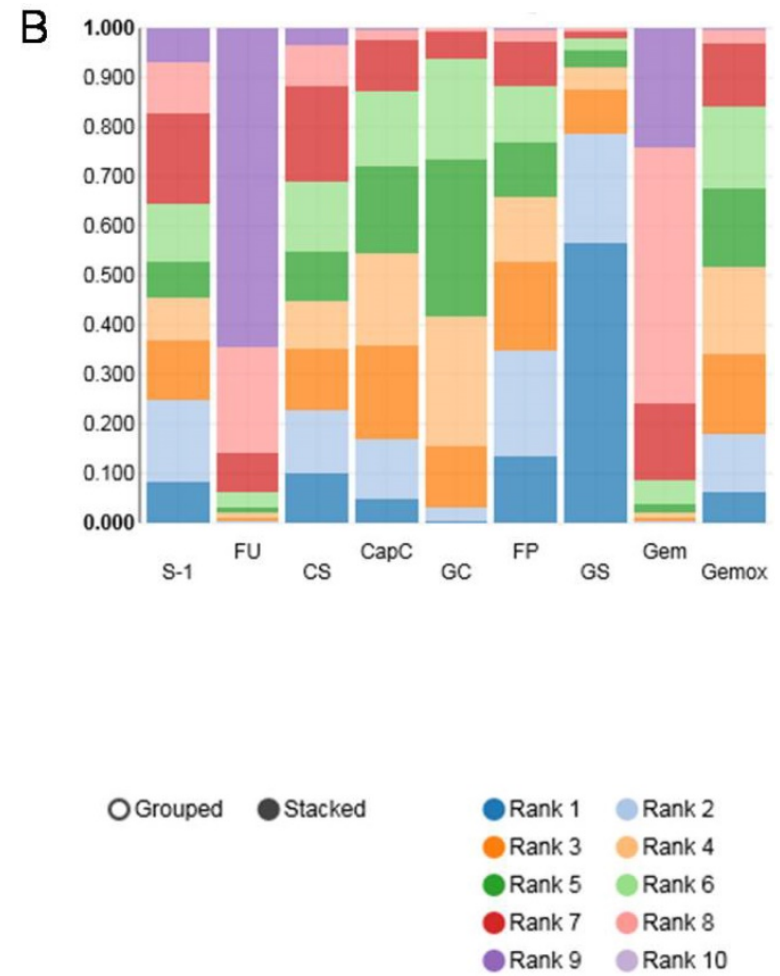

Figure 3. Ranking probability results of ORR (A), OS (B) and PFS (C). Rank 1 is best, rank $N$ is worst. 
Table 2. Network meta-analysis results for ORR, PFS and OS.

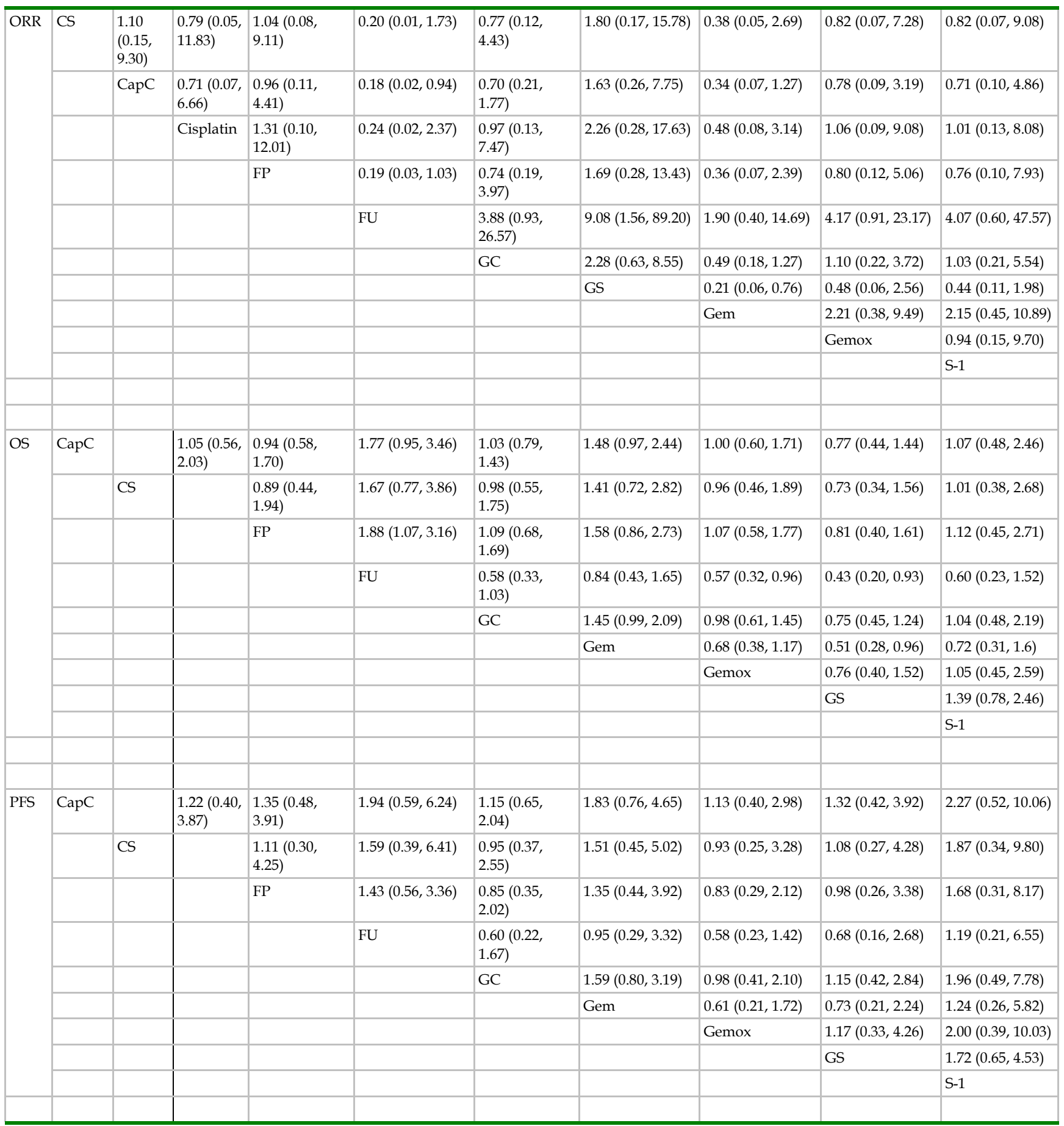

Note: Each cell gives the effect of the column-defining intervention relative to the row-defining intervention. The bold form means significant result.

Table 3. Network meta-analysis results for adverse events.

\begin{tabular}{|c|c|c|c|c|c|c|c|c|c|c|}
\hline \multirow[t]{4}{*}{ vomiting } & CS & $\begin{array}{l}6.95(0.12, \\
3.99 \mathrm{E}+02)\end{array}$ & & & $\begin{array}{l}3.32 \mathrm{E}+06 \\
(3.16, \\
3.12 \mathrm{E}+19)\end{array}$ & $\begin{array}{l}2.60(0.07 \\
1.14 \mathrm{E}+02)\end{array}$ & $\begin{array}{l}2.56(0.08 \\
8.80 \mathrm{E}+01)\end{array}$ & $\begin{array}{l}2.60(0.06 \\
1.59 \mathrm{E}+02)\end{array}$ & $\begin{array}{l}0.69(0.01 \\
7.99 \mathrm{E}+01)\end{array}$ & $\begin{array}{l}\text { throcopen } \\
\text { ia }\end{array}$ \\
\hline & $\begin{array}{l}0.00(0.00, \\
0.47)\end{array}$ & CapC & & & $\begin{array}{l}1.34(0.29, \\
6.90)\end{array}$ & $\begin{array}{l}0.38(0.01, \\
1.32 \mathrm{E}+01)\end{array}$ & $\begin{array}{l}0.38(0.01 \\
1.02 \mathrm{E}+01)\end{array}$ & $\begin{array}{l}0.39(0.01 \\
2.05 E+01)\end{array}$ & $\begin{array}{l}0.10(0.00, \\
9.61)\end{array}$ & \\
\hline & $\begin{array}{l}0.00(0.00, \\
2.16)\end{array}$ & $\begin{array}{l}1.49(0.01 \\
1.54 \mathrm{E}+02)\end{array}$ & FP & & & & & & & \\
\hline & $\begin{array}{l}0.00(0.00, \\
3.36)\end{array}$ & $\begin{array}{l}3.94(0.07, \\
1.95 \mathrm{E}+02)\end{array}$ & $\begin{array}{l}2.53(0.24 \\
3.87 \mathrm{E}+01)\end{array}$ & $\mathrm{FU}$ & & & & & & \\
\hline
\end{tabular}




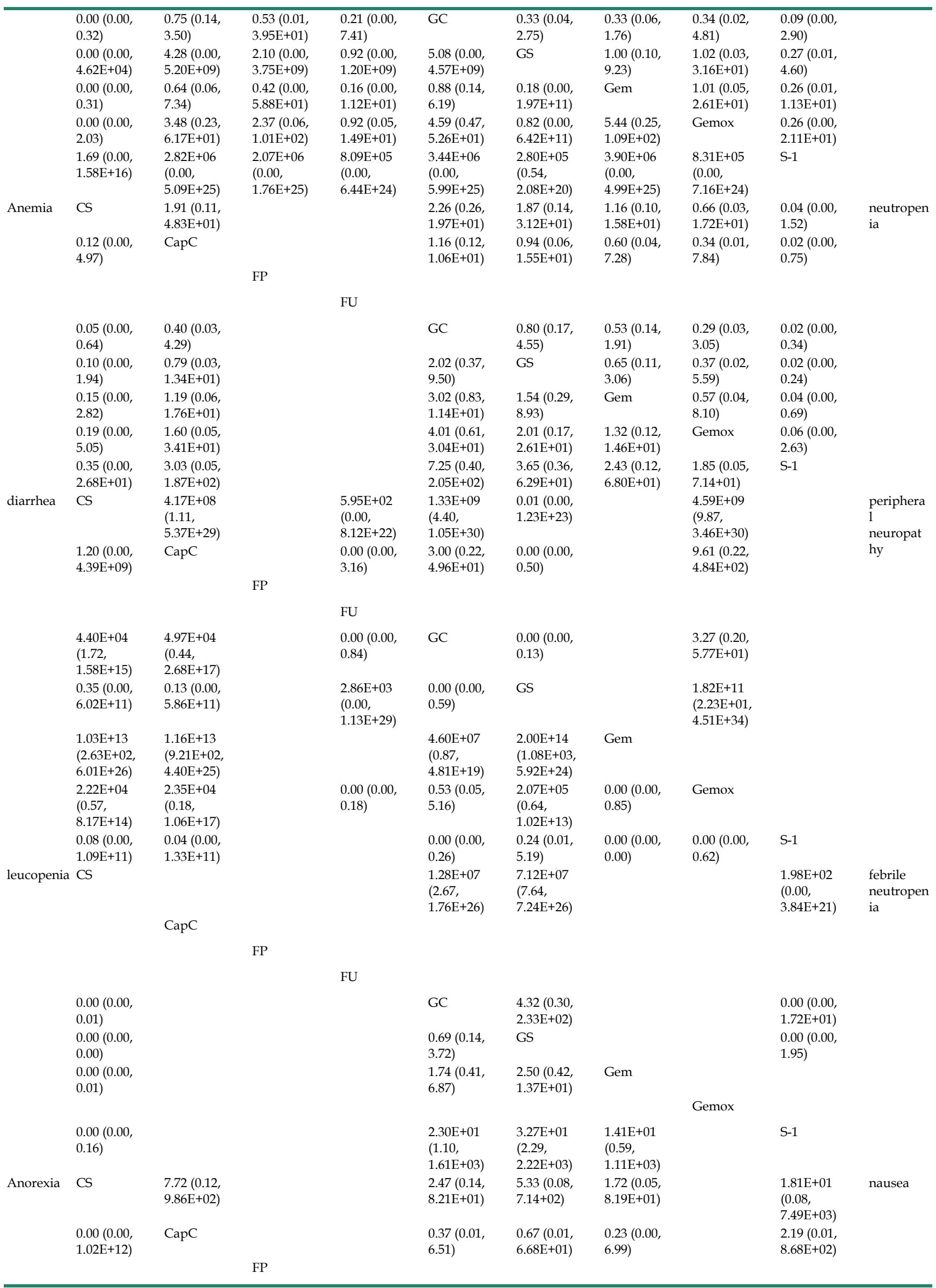




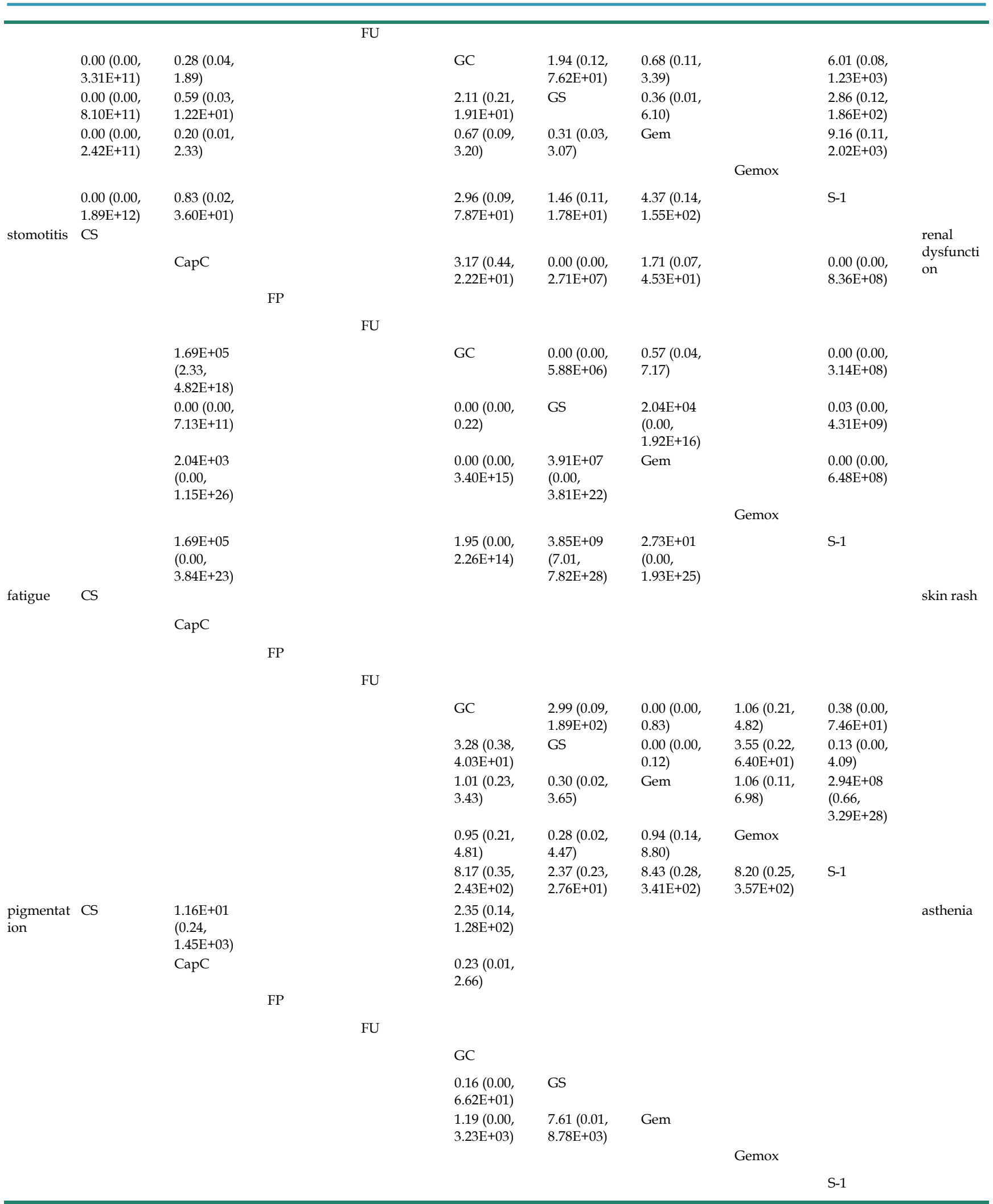



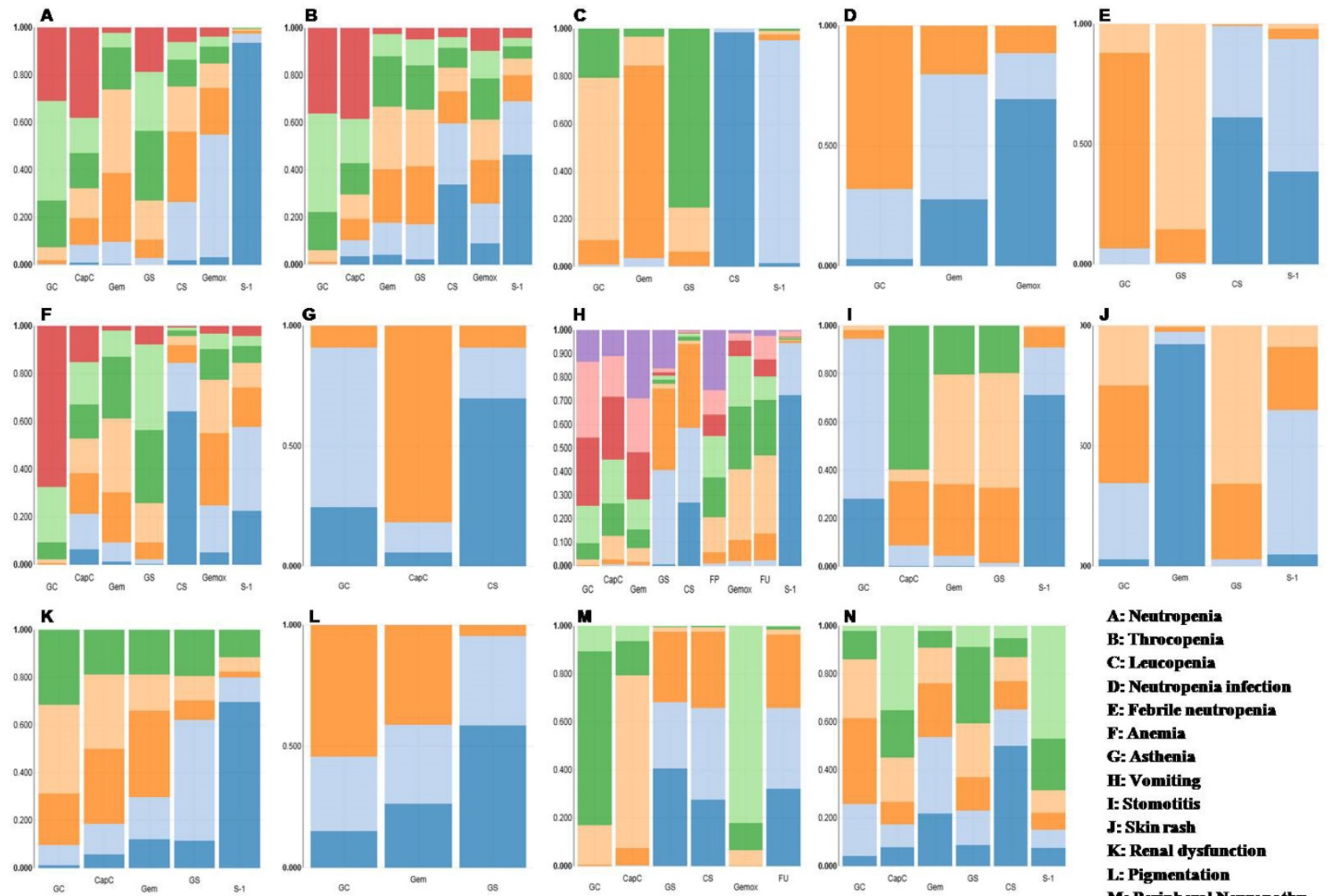

A: Neutropenia

B: Throcopenia C: Leucepenia

D: Neutropenia infection E: Febrile neutropenia

F: Anemia

G: Asthenia

H: Vomiting

I: Stomotitis

J: Skin rash

K: Renal dysfunction L: Pigmentation M: Peripheral Neuropathy N: Nausea
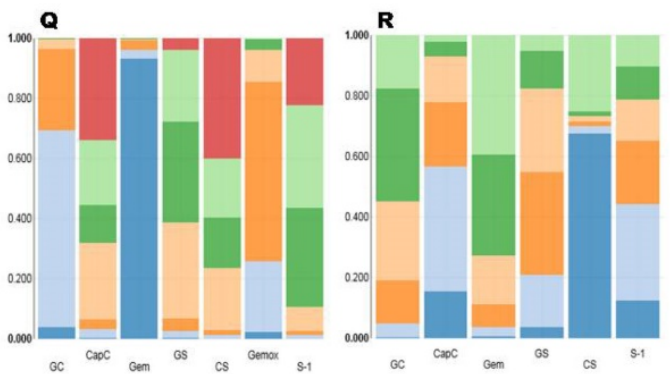

O: Hand-foet syndrome

P: Fatigure

Q: Diarnea

R: Anorexia

OGrouped Rank 1 Rank 2

- Stacked Rank 3 Rank 4

Rank 5 Rank 6

-Rank 7 Rank 8

0.000

$\propto$

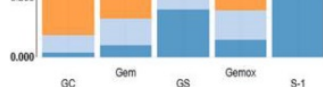

ac carc aem os cs Gemax

Figure 4. Ranking probability results of adverse events. Rank 1 is best, rank $N$ is worst.

\section{Discussion}

BTC is an aggressive cancer causing a high mortality around the world. One-year survival for intrahepati BTC was $25 \%$, decreasing to $8 \%$ and $5 \%$ at 3 and 5 years [11]. In extrahepatichepatic BTC survival less rapidly reduced from $48 \%$ at 1 year to $23 \%$ at 3 years [11]. For the majority of the patients who present with locally advanced or metastatic disease, systemic chemotherapy remains the mainstay treatment. To give valuable suggestions for chemotherapy regiments by comparing the efficacy and safety, we conducted this network meta-analysis based on 16 studies involving a total of 2245 individuals, with six drugs commonly used for ABTC.

This meta-analysis showed that doublet chemotherapy was more outstanding than mono therapy for efficacy in ORR, PFS and OS, but with higher risk of toxicity. According to ranking of treatments, Gem based therapies showed advantages in OS; fluoropyrimidines based regimens were associated with an increased ORR. And platinum compounds based chemotherapies may have PFS benefits. Overall, GS probably have some advantages in terms of efficacy (OS and ORR) in treating patients with ABTC.

S-1, an oral fluoropyrimidine derivative, has been widely used as one of the key drugs for the treatment of BTC. An ORR of $15 \%-20 \%$ and median OS of 7-9 month were obtained for S-1 monotherapy as the first-line treatment in patients with ABTC [12-14]. Because of its high anti-tumor activity, the combination therapy with gemcitabine, another crucial drug for ABTC, was tried and has shown promising results in several phase II trials $[15,16]$. 
Recently, a multicenter retrospective study compared GS and GC treatment in patients with ABTC, and demonstrated that GS and GC were similar with regard to their safety and efficacy [17]. Outcomes of this network analysis are consistent with the conclusion from previous clinical researches. On the other hand, patients treated with S-1 could have better compliance and higher quality of life than received cisplatin, with regard to its oral administration. Also, GS therapy can be continued until disease progression, although GC therapy should be terminated before progression if cumulative toxicity of cisplatin such as peripheral neuropathy or renal damage is observed [18]. Therefore, in addition to the standard recommendation (GC), GS may be worthy of consideration in clinical for the treatment of ABTC. Further evaluation should be given.

However, all the treatments assessed were not associated with surprising survival prospects compared with GC regiment, the standard chemotherapy for ABTC at present. As BTC is a heterogeneous group of tumors, a large amount of work has been recently accomplished to gain deeper insights into its biological mechanism [19]. Until now, no effective molecular biomarkers are currently available to predict response and survival outcome after treatment in ABTC patients. Targeted therapy for ABTC is still in its infancy and the experience of other successful cancers is borrowed. For example, oncogenic activation of EGFR and its downstream pathways, including KRAS, BRAF, PTEN, PIK3CA et al, are correlated with responsiveness to anti-EGFR therapies [20, 21]. In colorectal cancer, it has been showed that KRAS mutation precludes any therapeutic benefit from anti-EGFR antibodies (cetuximab and panitumumab). And EGFR mutation point benefits from erlotinib in lung cancer. Although ABTCs have a spectrum of mutations in EGFR signaling pathway, the concept above has not yet been confirmed [22, 23]. VEGF, one of the main growth factors regulating angiogenesis, is over expressed in $40-75 \%$ of BTC. However, sorafenib or cediranib combined with conventional chemotherapy does not exceed the outcome of patients treated with chemotherapy alone [23-27]. We hold the opinion that the use of Next-generation sequencing (NGS) and the establishment of molecular classification could make the breakthrough of targeted therapy for BTC possible. Further prospective studies are needed.

There are some limitations in our analysis. First, given the rarity of this disease, samples in some of the clinical studies included in this network analysis were small and not randomized. Second, limited by the absence of some raw data, the subgroup analyses for location of the primary tumor, disease status and metastatic sites et al were not conducted. On the other hand, classical risk factors for biliary tract cancer include chronic cholestatic disease (primary biliary cirrhosis) and chronic inflammation of the biliary tract (calculi, malformations, Caroli disease, hamartoma, chronic parasitic or bacterial infections). However, the majority of patients develop biliary tract cancer in the absence of identifiable risk factors clinically [28]. The interaction between risk factors and chemotherapy in patients with biliary tract cancer is not described and analyzed in all literatures included in this network meta-analysis. Admittedly, as a re-analysis of the relevant data, it is difficult to describe the causation elements of these patients with the disease. Therefore, we did not carry out a subgroup analysis of the incentives here, too. Third, not all adverse events were calculated among the ten therapeutic regimens. Fourth, during the process of literature review and data extraction, we noted that there were various standards of liver dysfunction. This inconsistency in evaluating criteria can skew results, so we did not analyse this common adverse effect here.

In conclusion, a regimen with an excellent combination of efficacy and safety remains to be discovered. Physicians should discuss with the patients the different options outlining potential benefit and toxicity since no clear evidence of an approach of choice can be produced in the existing chemotherapies.

\section{Competing Interests}

The authors have declared that no competing interest exists.

\section{References}

1. Bridgewater JA, Goodman KA, Kalyan A, Mulcahy MF. Biliary Tract Cancer: Epidemiology, Radiotherapy, and Molecular Profiling. Am Soc Clin Oncol Educ Book. 2016; 35: e194-203.

2. Razumilava N, Gores GJ. Cholangiocarcinoma. Lancet. 2014; 383: 2168-79.

3. Groot KB, Fong Y. Outcomes in biliary malignancy. J Surg Oncol. 2014; 110: 585-91.

4. Edge SB, Compton CC. The American Joint Committee on Cancer: the 7th edition of the AJCC cancer staging manual and the future of TNM. Ann Surg Oncol. 2010; 17: 1471-4.

5. Esnaola NF, Meyer JE, Karachristos A, Maranki JL, Camp ER, Denlinger CS. Evaluation and management of intrahepatic and extrahepatic cholangiocarcinoma. Cancer. 2016; 122: 1349-69.

6. Park JO, Oh DY, Hsu C, Chen JS, Chen LT, Orlando M, et al. Gemcitabine Plus Cisplatin for Advanced Biliary Tract Cancer: A Systematic Review. Cancer Res Treat. 2015; 47: 343-61.

7. Bridgewater J, Lopes A, Palmer D, Cunningham D, Anthoney A, Maraveyas $\mathrm{A}$, et al. Quality of life, long-term survivors and long-term outcome from the ABC-02 study. Br J Cancer. 2016; 114: 965-71.

8. Marks EI, Yee NS. Molecular genetics and targeted therapeutics in biliary tract carcinoma. World J Gastroenterol. 2016; 22: 1335-47.

9. Higgins JP, Thompson SG, Deeks JJ, Altman DG. Measuring inconsistency in meta-analyses. BMJ. 2003; 327: 557-60.

10. Bowden J, Tierney JF, Copas AJ, Burdett S. Quantifying, displaying and accounting for heterogeneity in the meta-analysis of RCTs using standard and generalised Q statistics. BMC Med Res Methodol. 2011; 11: 41.

11. Mosconi S, Beretta GD, Labianca R, Zampino MG, Gatta G, Heinemann V. Cholangiocarcinoma. Crit Rev Oncol Hematol. 2009; 69: 259-70.

12. Sasaki T, Isayama H, Yashima $Y$, Yagioka H, Kogure H, Arizumi T, et al. S-1 monotherapy in patients with advanced biliary tract cancer. Oncology. 2009; 77: 71-4. 
13. Furuse J, Okusaka T, Boku N, Ohkawa S, Sawaki A, Masumoto T, et al. S-1 monotherapy as first-line treatment in patients with advanced biliary tract cancer: a multicenter phase II study. Cancer Chemother Pharmacol. 2008; 62: 849-55.

14. Morizane C, Okusaka T, Mizusawa J, Takashima A, Ueno M, Ikeda M, et al. Randomized phase II study of gemcitabine plus S-1 versus S-1 in advanced biliary tract cancer: a Japan Clinical Oncology Group trial (JCOG 0805). Cancer Sci. 2013; 104: 1211-6.

15. Kim HS, Kim HY, Zang DY, Oh HS, Jeon JY, Cho JW, et al. Phase II study of gemcitabine and S-1 combination chemotherapy in patients with metastatic biliary tract cancer. Cancer Chemother Pharmacol. 2015; 75: 711-8.

16. Sasaki $\mathrm{T}$, Isayama $\mathrm{H}$, Nakai $\mathrm{Y}$, Ito $\mathrm{Y}, \mathrm{Kogure} H$, Togawa $\mathrm{O}$, et al. Multicenter, phase II study of gemcitabine and S-1 combination chemotherapy in patients with advanced biliary tract cancer. Cancer Chemother Pharmacol. 2010; 65: 1101-7.

17. Takahara N, Isayama H, Nakai Y, Sasaki T, Ishigaki K, Saito K, et al. Gemcitabine and S-1 versus gemcitabine and cisplatin treatment in patients with advanced biliary tract cancer: a multicenter retrospective study. Invest New Drugs. 2017; 35: 269-76.

18. Mizusawa J, Morizane C, Okusaka T, Katayama H, Ishii H, Fukuda H, et al. Randomized Phase III study of gemcitabine plus S-1 versus gemcitabine plus cisplatin in advanced biliary tract cancer: Japan Clinical Oncology Group Study (JCOG1113, FUGA-BT). Jpn J Clin Oncol. 2016; 46: 385-8.

19. Verlingue L, Hollebecque A, Boige V, Ducreux M, Malka D, Ferte C. Matching genomic molecular aberrations with molecular targeted agents: Are biliary tract cancers an ideal playground. Eur J Cancer. 2017; 81: 161-73.

20. Chen JS, Hsu C, Chiang NJ, Tsai CS, Tsou HH, Huang SF, et al. A KRAS mutation status-stratified randomized phase II trial of gemcitabine and oxaliplatin alone or in combination with cetuximab in advanced biliary tract cancer. Ann Oncol. 2015; 26: 943-9.

21. Kim ST, Jang KT, Lee J, Jang HM, Choi HJ, Jang HL, et al. Molecular Subgroup Analysis of Clinical Outcomes in a Phase 3 Study of Gemcitabine and Oxaliplatin with or without Erlotinib in Advanced Biliary Tract Cancer. Transl Oncol. 2015; 8: 40-6.

22. Malka D, Cervera P, Foulon S, Trarbach T, de la Fouchardiere C, Boucher E, et al. Gemcitabine and oxaliplatin with or without cetuximab in advanced biliary-tract cancer (BINGO): a randomised, open-label, non-comparative phase 2 trial. Lancet Oncol. 2014; 15: 819-28.

23. Leone F, Marino D, Cereda S, Filippi R, Belli C, Spadi R, et al. Panitumumab in combination with gemcitabine and oxaliplatin does not prolong survival in wild-type KRAS advanced biliary tract cancer: A randomized phase 2 trial (Vecti-BIL study). Cancer. 2016; 122: 574-81.

24. Santoro A, Gebbia V, Pressiani T, Testa A, Personeni N, Arrivas BE, et al. A randomized, multicenter, phase II study of vandetanib monotherapy versus vandetanib in combination with gemcitabine versus gemcitabine plus placebo in subjects with advanced biliary tract cancer: the VanGogh study. Ann Oncol. 2015; 26: 542-7.

25. Moehler M, Maderer A, Schimanski C, Kanzler S, Denzer U, Kolligs FT, et al. Gemcitabine plus sorafenib versus gemcitabine alone in advanced biliary tract cancer: a double-blind placebo-controlled multicentre phase II AIO study with biomarker and serum programme. Eur J Cancer. 2014; 50: 3125-35.

26. Sahu S, Sun W. Targeted therapy in biliary tract cancers-current limitations and potentials in the future. J Gastrointest Oncol. 2017; 8: 324-36.

27. Valle JW, Wasan H, Lopes A, Backen AC, Palmer DH, Morris K, et al. Cediranib or placebo in combination with cisplatin and gemcitabine chemotherapy for patients with advanced biliary tract cancer (ABC-03): a randomised phase 2 trial. Lancet Oncol. 2015; 16: 967-78.

28. Blechacz B. Cholangiocarcinoma: Current Knowledge and New Developments. Gut Liver. 2017; 11: 13-26. 